\title{
Identification of early biological changes in palmitate-treated isolated human islets
}

\author{
Ernest Sargsyan ${ }^{1,2^{*}}$, Jing Cen ${ }^{1}$, Kirsten Roomp ${ }^{3}$, Reinhard Schneider ${ }^{3}$ and Peter Bergsten ${ }^{1}$
}

\begin{abstract}
Background: Long-term exposure to elevated levels of free fatty acids (FFAs) is deleterious for beta-cell function and may contribute to development of type 2 diabetes mellitus (T2DM). Whereas mechanisms of impaired glucosestimulated insulin secretion (GSIS) in FFA-treated beta-cells have been intensively studied, biological events preceding the secretory failure, when GSIS is accentuated, are poorly investigated. To identify these early events, we performed genome-wide analysis of gene expression in isolated human islets exposed to fatty acid palmitate for different time periods.

Results: Palmitate-treated human islets showed decline in beta-cell function starting from day two. Affymetrix Human Transcriptome Array 2.0 identified 903 differentially expressed genes (DEGs). Mapping of the genes onto pathways using KEGG pathway enrichment analysis predicted four islet biology-related pathways enriched prior but not after the decline of islet function and three pathways enriched both prior and after the decline of islet function. DEGs from these pathways were analyzed at the transcript level. The results propose that in palmitate-treated human islets, at early time points, protective events, including up-regulation of metallothioneins, tRNA synthetases and fatty acid-metabolising proteins, dominate over deleterious events, including inhibition of fatty acid detoxification enzymes, which contributes to the enhanced GSIS. After prolonged exposure of islets to palmitate, the protective events are outweighed by the deleterious events, which leads to impaired GSIS.

Conclusions: The study identifies temporal order between different cellular events, which either promote or protect from beta-cell failure. The sequence of these events should be considered when developing strategies for prevention and treatment of the disease.
\end{abstract}

\section{Background}

Elevated levels of circulating free fatty acids is one of the major factors implicated in alteration of insulin secretion in obese individuals [1]. In many of these individuals initial hyperinsulinemia is followed by later reduction of insulin secretion and development of type 2 diabetes mellitus (T2DM) $[2,3]$. These clinical observations are supported by in vitro studies which show that a short-term exposure to fatty acids potentiates insulin secretion whereas a long-term exposure exerts deleterious effects [4-6].

Current treatment strategies aim to restore beta-cell function when functional decline is already evident.

\footnotetext{
* Correspondence: ernest.sargsyan@mcb.uu.se

${ }^{1}$ Department of Medical Cell Biology, Uppsala University, Box 571, 75123 Uppsala, Sweden

${ }^{2}$ Molecular Neuroscience Group, Institute of Molecular Biology, National

Academy of Sciences, 0014 Yerevan, Armenia

Full list of author information is available at the end of the article
}

However, accumulating evidence indicate that therapeutic intervention at later stages when beta-cell function is already lost is inefficient and does not diminish the risk of T2DM [7]. Our observation that intracellular insulin content in fatty acid-treated isolated human islets is declined prior to the failure in insulin secretion suggests that deleterious mechanisms in fatty acid-exposed human islets are already activated at early time points when islets still hypersecrete insulin [4]. Therefore, it is desirable to identify such early mechanisms in order to prevent or reverse the disease by intervening at early stages when beta-cell function is not yet lost and intracellular changes have not passed a point of no return.

Mechanisms that are implicated in the long-term effects of fatty acids on beta cells have been intensively investigated. These mechanisms include alterations in mitochondrial function [8, 9], ER stress response [10], generation of ceramide species $[11,12]$, impairment of

(c) The Author(s). 2018 Open Access This article is distributed under the terms of the Creative Commons Attribution 4.0 International License (http://creativecommons.org/licenses/by/4.0/), which permits unrestricted use, distribution, and 
exocytotic machinery [13] and disturbances in GPR40 signalling $[4,14]$. In contrast, events occurring prior to the failure of beta cells, when islets hypersecrete insulin, are much less understood.

The aim of our study was to identify the biological events preceding the failure of beta cells in fatty acid-treated human islets. The aim was addressed by using genome-wide analysis of gene expression in isolated human islets exposed to fatty acid palmitate for various time periods (Fig. 1).

\section{Methods}

\section{Culture of isolated human islets}

Human islets were obtained from brain-dead otherwise healthy individuals from the Islet Prodo Lab Inc. (Irvine, CA, USA). The donors contributing islets to this study were two male donors aged 59 and 66 and three female donors aged 34, 39 and 48. Purity of islets varied between 85 and 90\%. Islets were shipped in PIM (T) medium (Islet Prodo Lab Inc.) within 2-4 days after isolation. Upon arrival, islets were cultured in CMRL 1066 medium containing $5.5 \mathrm{mM}$ glucose and supplemented with $10 \%$ FBS.

\section{Free fatty acid treatment of isolated human islets}

Palmitate was prepared as $100 \mathrm{mM}$ stock solution in $50 \%$ ethanol. The stock solution was diluted in culture medium to $0.5 \mathrm{mM}$ concentration and complexed with $0.5 \mathrm{mM}$ free fatty-acid free bovine serum albumin (BSA) for $30 \mathrm{~min}$ at $37^{\circ} \mathrm{C}$. Human islets were cultured in the absence and presence of palmitate for 4 and $12 \mathrm{~h}$ and 1 , 2 and 7 days. Experiments were designed in a way that all treatments were terminated at day 7 . Prior the treatments islets were cultured under control conditions and culture media were changed daily. After treatment, islets were picked up individually (to avoid non-islet structures) for glucose-stimulated insulin secretion, insulin content, and transcriptomics analyses.

\section{Insulin secretion and insulin content of human islets}

After treatment, 15-20 human islets were picked individually and placed into a perifusion chamber. Islets were perifused at $37^{\circ} \mathrm{C}$ with a buffer $(\mathrm{pH}=7.4)$ containing $125 \mathrm{mM} \mathrm{NaCl}, 5.9 \mathrm{mM} \mathrm{KCl}, 1.2 \mathrm{mM} \mathrm{MgCl}_{2}, 1.3$ $\mathrm{mM} \mathrm{CaCl} 2,25 \mathrm{mM}$ HEPES and 1\% $(w / v)$ fatty acid free BSA (fraction V; Boehringer Mannheim $\mathrm{GmbH}$ ). The perifusion rate was $170 \mu \mathrm{l} / \mathrm{min}$. During the first hour islets were perifused with a buffer containing $2 \mathrm{mM}$ glucose, which was followed by a 20 min perifusion with a buffer containing $20 \mathrm{mM}$ glucose. Perifusates were collected at $-15,-10,-5,0,2,4,6,10,15,20 \mathrm{~min}$ to measure the amounts of secreted insulin. At $0 \mathrm{~min}$ the glucose concentration was raised from 2 to $20 \mathrm{mM}$. After perifusion, islets were washed with Dulbecco's

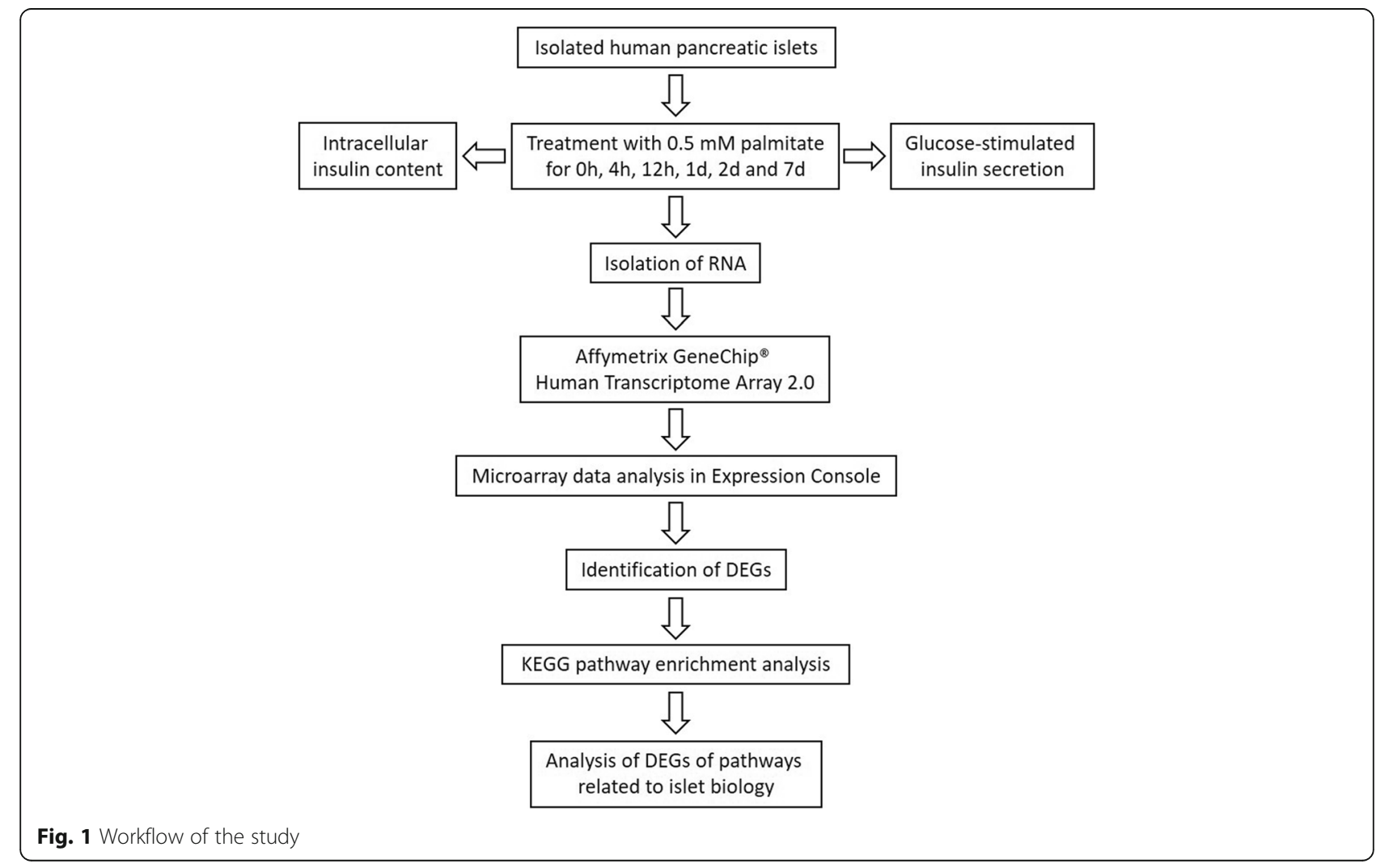


phosphate buffered saline (DPBS) and lysed in DPBS buffer containing $1 \%$ Triton X100 and $0.4 \%$ protease inhibitor cocktail (both obtained from Sigma Aldrich). Lysates were used for measurements of insulin and protein content. Insulin was determined by a competitive ELISA, as previously described [15]. For each perifusion, insulin secretory rate at stimulatory $(20 \mathrm{mM})$ glucose was normalized to total protein. Insulin secretion was expressed as a ratio between insulin secretory rate at stimulatory glucose from treated islets and insulin secretory rate at stimulatory glucose from untreated islets for each donor. Insulin content was normalized to islet protein and expressed as fold control.

\section{Preparation of mRNA}

After treatment, 50 human islets were individually collected and washed with PBS tree times. mRNA was isolated using mRNA isolation kit from Macherey-Nagel (Duren, Germany) according to the manufacturer's instructions. RNA concentration was measured with ND-1000 spectrophotometer (NanoDrop Technologies, Wilmington, DE) and RNA quality was evaluated using the Agilent 2100 Bioanalyzer system (Agilent Technologies Inc., Palo Alto, CA).

\section{Microarray expression analysis}

Total RNA, 100 nanograms from each sample, was used to generate amplified and biotinylated sense-strand cDNA from the entire expressed genome according to the GeneChip ${ }^{\circ}$ WT PLUS Reagent Kit User Manual (P/ N 703174 Rev. 1 Affymetrix Inc., Santa Clara, CA). GeneChip ${ }^{\circ}$ ST Arrays (GeneChip Human Transcriptome Array (HTA) 2.0) were hybridized for $16 \mathrm{~h}$ in a $45^{\circ} \mathrm{C}$ incubator, rotated at $60 \mathrm{rpm}$. According to the GeneChip ${ }^{\circ}$ Expression Wash, Stain and Scan Manual (PN 702731 Rev. 3, Affymetrix Inc., Santa Clara, CA) the arrays were then washed and stained using the Fluidics Station 450 and finally scanned using the GeneChip Scanner 3000 7G. The HTA array covers almost 68,000 genes of which 27,000 are annotated.

\section{Microarray data analysis}

The raw data was normalized in Expression Console, provided by Affymetrix (http://www.affymetrix.com), using the robust multi-array average (RMA) method as previously described $[16,17]$. Genes with a more than 1.3-fold change after palmitate exposure compared to untreated islets were defined as differentially expressed (DEGs).

\section{KEGG pathway enrichment analysis}

Differentially expressed genes were selected for bioinformatics analysis. KEGG (Kyoto Encyclopedia of Genes and Genomes) pathway over-representation analysis was done using ConsensusPathDB (http://consensuspathdb.org/) [18]. In the further analysis, DEGs in the pathways of interest were manually annotated using literature survey and UniProt database.

\section{Statistical analysis}

Results of glucose-stimulated insulin secretion (GSIS) and insulin content are presented as means \pm SEM. Gene's expression is presented as mean $\pm \mathrm{SD}$. Due to high variability between islets and limited resources to increase the sample size, we increased the validity of the results by normalizing each experiment to its own control. Statistical significance was evaluated by using one-way ANOVA with Dunnett's multiple comparison test. $p<0.05$ was considered statistically significant.

KEGG pathway over-representation analysis in ConsensusPathDB was carried out using input gene lists that were compared to functional modules derived from KEGG pathway definitions. A $p$-value cut-off of $<0.01$ and a minimum overlap with the input list of two genes were used. The calculated $p$-value reflects the significance of the observed overlap between an input gene list and a module's members, as compared to random expectations. Therefore, small $p$-values indicate that of the genes in the input list, more are present in a module (pathway) than would be expected by chance alone and this may indicate dysregulated pathways [19].

\section{Results}

GSIS and insulin content of human islets exposed to palmitate

GSIS and insulin content was determined in isolated human islets exposed to palmitate for 0,4 and $12 \mathrm{~h}$ and 1 , 2 and 7 days. After 4 h, GSIS was not changed compared with control islets. After a longer exposure period, GSIS gradually increased reaching the maximal level after 1 day and then decreased to $70 \%$ of control level after 7 days (Figs. 2a, b). Intracellular insulin content was not changed up to 1-day culture with palmitate but gradually decreased to approximately 75 and 30\% of control level after 2 and 7 days, respectively (Fig. 2c).

\section{Transcriptome of human islets exposed to palmitate}

To delineate mechanisms for these time-dependent changes in GSIS and insulin content we performed a transcriptomics analysis of human islets exposed to palmitate for 0,4 and $12 \mathrm{~h}$ and 1,2 and 7 days. The genes were defined as differentially expressed if they were changed more than 1.3-fold after palmitate treatment. Using this cut-off we found that out of 27,000 transcripts 903 unique genes were differentially expressed at least in one of the culture periods. The number of DEGs increased with the exposure time 


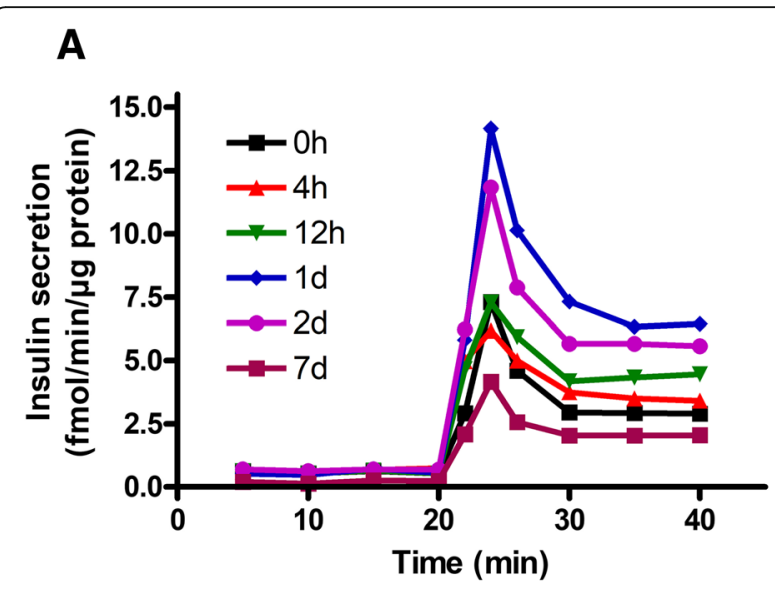

B

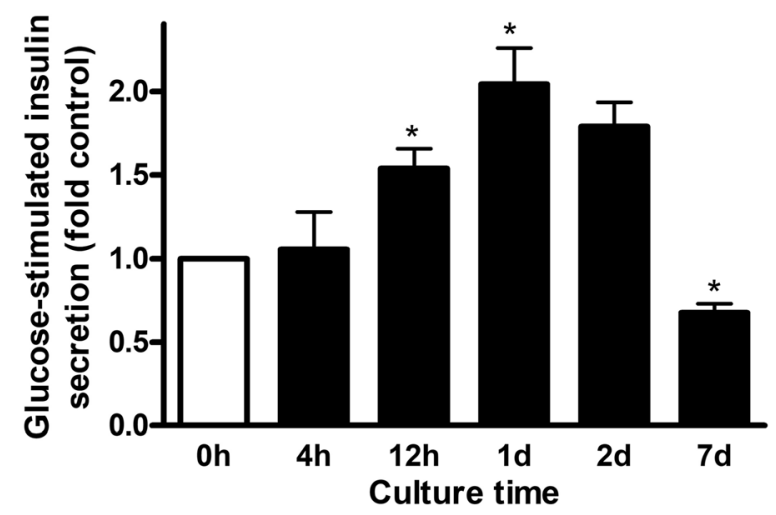

C

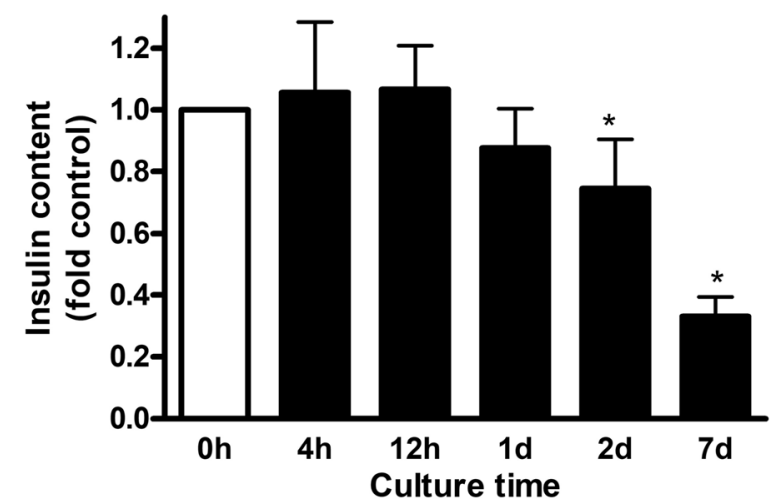

Fig. 2 GSIS and intracellular insulin content in isolated human islets exposed to palmitate for 0,4 and $12 \mathrm{~h}$ and 1, 2 and 7 days. After culture, islets were perifused with $2 \mathrm{mM}$ glucose followed by 20 min perifusion with 20 mM glucose. Panel a. Representative graphs of dynamic insulin secretion from one donor. Panel $\mathbf{b}$. Insulin secretory rate at stimulatory (20 mM) glucose was normalized to total protein. Insulin secretion was expressed as a ratio between insulin secretory rate at stimulatory glucose from treated islets and insulin secretory rate at stimulatory glucose from untreated islets for each donor. Panel $\mathbf{c}$. Islets were subsequently lysed and insulin content measured. Insulin content after treatments was normalized to total protein content and expressed as fold insulin content in untreated islets. Results are means of 5 donors \pm SEM. ${ }^{*} p<0.05$ vs control
(Fig. 3a). It was 80 after 4 h, 142 after 12 h, 167 after 1 day, 259 after 2 days and 759 after 7 days.

Further analysis of the DEGs demonstrated that the percentages of up- and down-regulated genes also changed with the exposure time (Fig. 3a). Whereas the proportion of down-regulated genes was only $10 \%$ after 4 and $12 \mathrm{~h}$ (8 genes and 15 genes, respectively), the percentage rose to $35 \%$ (58 genes) after 1 day, 63\% (163 genes) after 2 days and 68\% (517 genes) after 7 days.

Next, we followed DEGs over time (Fig. 3b). We found that $\sim 300$ genes were differentially expressed prior to the decline of beta-cell function, up to 1-day of palmitate exposure. Of those, 36 DEGs (40\%) after $4 \mathrm{~h}, 64$ DEGs (55\%) after $12 \mathrm{~h}$, and 49 DEGs (60\%) after 1 day
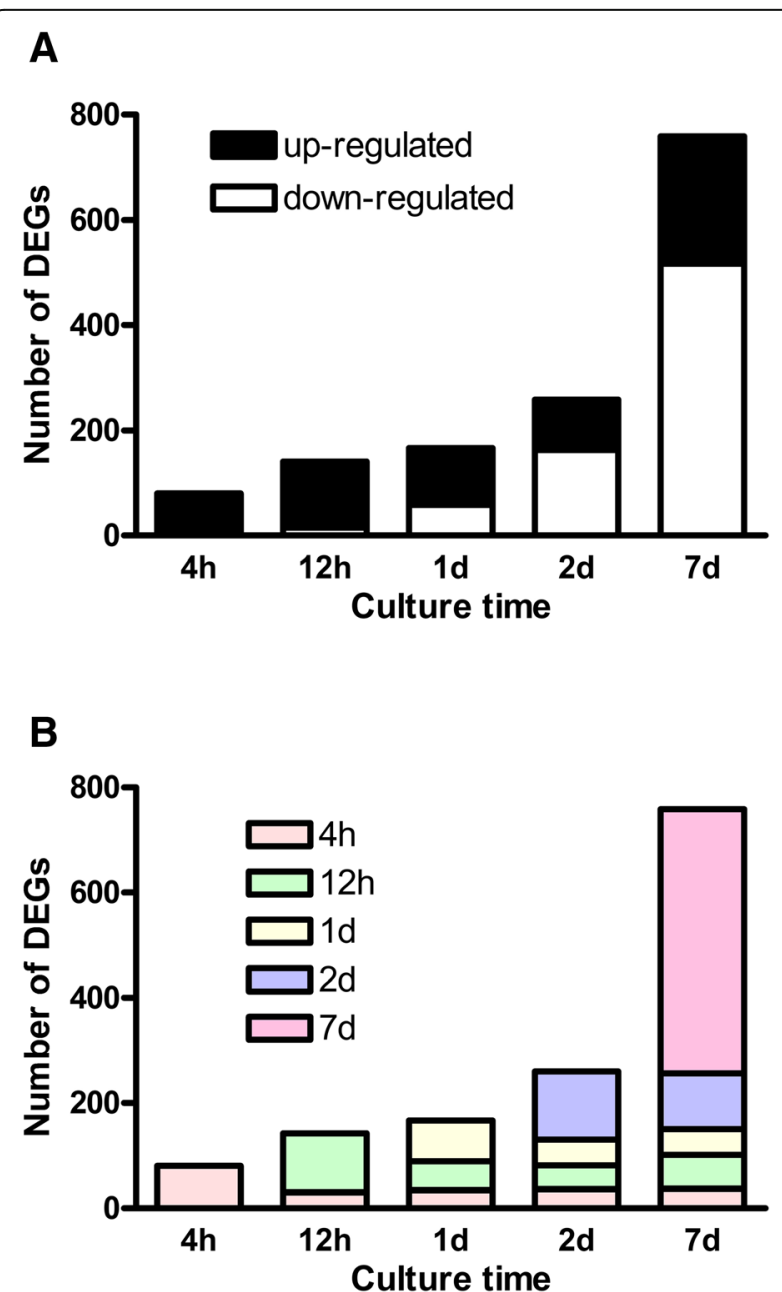

Fig. 3 Transcriptome of isolated human islets exposed to palmitate for 0,4 and $12 \mathrm{~h}$ and 1,2 and 7 days. Transcriptomics analysis was performed by using GeneChip ${ }^{\circledR}$ Human Transcriptome Array 2.0 from Affymetrix. Genes were defined as differentially expressed if they changed more than 1.3-fold after palmitate treatment with the significance level of $p \leq 0.05$. Panel a. Number of up- and downregulated genes. Panel $\mathbf{b}$. Each color reflects DEGs that appear for the first time at a certain culture duration. Results are from 5 donors 
were differentially expressed also after 7 days. Interestingly, only 4 genes, all encoding members of aldo-keto reductase family 1 (B10, B15, C1 and C2), were changed in opposite directions over time. These genes were up-regulated after $12 \mathrm{~h}$ but down-regulated after 7 days of palmitate exposure i.e. in a similar way that was observed for GSIS.

\section{KEGG pathway enrichment analysis of differentially expressed genes}

Next, we mapped all the DEGs onto pathways using KEGG pathway enrichment analysis to obtain an overview of biological events in human islets during fatty acid exposure. The analysis predicted 56 pathways significantly enriched at least in one culture duration (Additional file 1: Table S1). Pathways enriched at each culture time points are listed separately (Additional file 2: Tables S2, Additional file 3: Table S3, Additional file 4: Table S4, Additional file 5: Table S5, Additional file 6: Table S6). Among these pathways we selected 15 related to islet biology (Fig. 4). To understand the early biological events during palmitate exposure, we have focused on those pathways which were enriched prior to the decline of islet function i.e. at 4 and $12 \mathrm{~h}$ and 1 day of palmitate exposure. Eight pathways were enriched already at these early time points. Of those, the four pathways, "Mineral absorption", "Aminoacyl-tRNA biosynthesis", "PPAR signaling pathway" and "Adipocytokine signaling pathway", were enriched only prior to the decline of islet function. The three pathways, "Metabolism of xenobiotics by P450", "Fatty acid degradation" and "Glycolysis/gluconeogenesis", were enriched both before and after the decline of islet function and the pathway "TNF signalling pathway" was enriched after 1 and 2 days of exposure to palmitate but not at other time points. The remaining seven pathways were enriched only after 2 and/or 7 days of palmitate exposure. Expression levels of the DEGs were further detailed at the different culture time points for the eight selected pathways (Table 1).

\section{Discussion}

\section{The study approach}

Elevated levels of free fatty acids are one of the major factors affecting insulin secretion from beta-cells [20]. Measurements of GSIS and intracellular insulin content in the current study demonstrated that up to 1 day's exposure to palmitate islet beta-cells enhance insulin secretion whereas longer exposure to palmitate leads to a degranulation and to a gradual decline in beta-cell secretory activity. In patients, when the capacity of islets to synthesize and to secrete insulin is diminished to a certain extent, T2DM develops.

In order to protect beta-cells from decline in the function it is important to identify early biological events triggered by the fatty acid exposure and to distinguish whether these pathways are protective/adaptive or deleterious. Such knowledge would provide an opportunity to develop strategies to reverse islet dysfunction and overt T2DM by preventing the negative developments and promoting adaptive processes [21].

To address the issue we identified gene signatures in fatty acid-treated human islets prior to and after the functional decline by combining transcriptomics and bioinformatics approaches. Previously, "omics" approaches helped to identify novel genes and metabolic pathways involved in palmitate-induced beta-cell dysfunction and death. A transcriptomics study on human islets identified 1325 genes differentially expressed after the long-term exposure to palmitate [22]. These DEGs belonged to functional categories "Beta-cell key transcription factors", "ER stress response", "Beta-cell signal transduction", "ATP production", "Metabolism" etc. [22]. Another transcriptomics study on human islets identified 1860 DEGs which were classified into 14 KEGG pathways including "Metabolic pathways", "Glycolysis/ Gluconeogenesis", "Fatty acid metabolism" and "Maturity onset diabetes of the young" [23]. Combination of proteomics and lipidomics approaches in our recent study showed that the elevated cholesterol and lipid biosynthesis, altered autocrine insulin signaling and decreased insulin granule maturation may play an

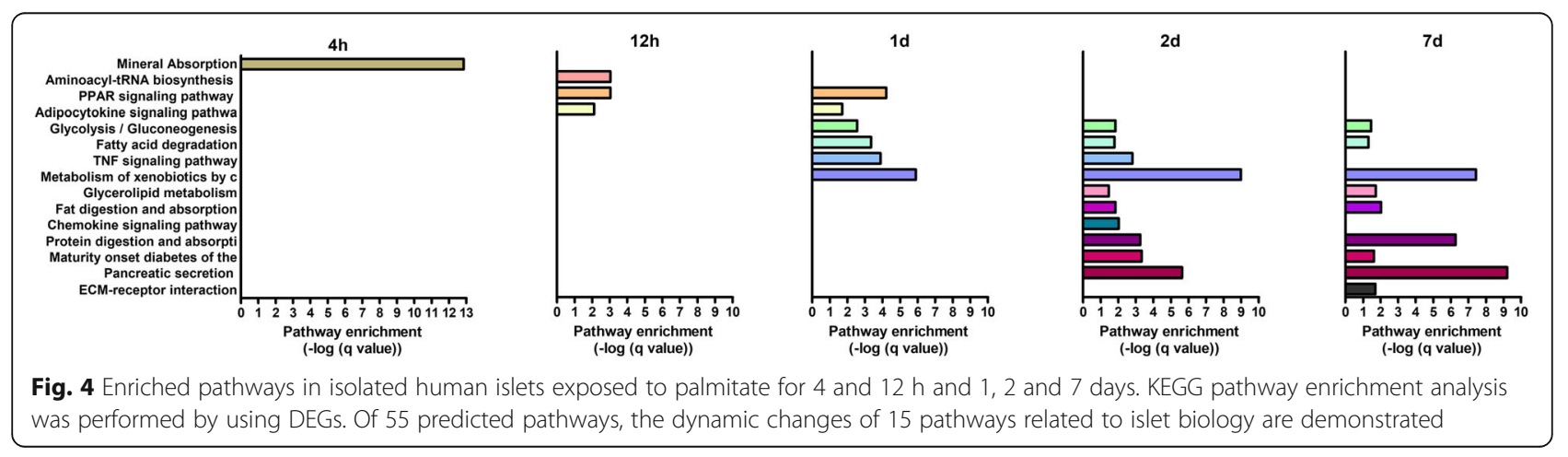


Table 1 Expression profile of genes from the enriched pathways over time (fold untreated \pm S.D., $n=5$ )

\begin{tabular}{|c|c|c|c|c|c|c|c|}
\hline Pathway name & $\begin{array}{l}\text { Gene } \\
\text { symbol }\end{array}$ & Gene name & $4 \mathrm{~h}$ & $12 \mathrm{~h}$ & $1 d$ & $2 d$ & $7 d$ \\
\hline \multirow[t]{11}{*}{ Mineral absorption } & MT1F & metallothionein $1 \mathrm{~F}$ & $\begin{array}{l}2.18 \pm \\
0.56^{*}\end{array}$ & $1.27 \pm 0.32$ & $1.36 \pm 0.42$ & $1.4 \pm 0.33$ & $1.41 \pm 0.58$ \\
\hline & MT2A & metallothionein 2A & $\begin{array}{l}2.19 \pm \\
0.37^{*}\end{array}$ & $1.08 \pm 0.31$ & $1.23 \pm 0.42$ & $1.32 \pm 0.48$ & $1.25 \pm 0.49$ \\
\hline & MT1L & $\begin{array}{l}\text { metallothionein } 1 \mathrm{~L} \text { (gene/ } \\
\text { pseudogene) }\end{array}$ & $2.2 \pm 0.45^{*}$ & $1.21 \pm 0.29$ & $1.26 \pm 0.23$ & $1.25 \pm 0.29$ & $1.17 \pm 0.26$ \\
\hline & MT1A & metallothionein $1 \mathrm{~A}$ & $\begin{array}{l}2.34 \pm \\
0.52^{*}\end{array}$ & $1.11 \pm 0.27$ & $1.21 \pm 0.2$ & $1.23 \pm 0.33$ & $1.2 \pm 0.38$ \\
\hline & MT1E & metallothionein $1 \mathrm{E}$ & $\begin{array}{l}2.58 \pm \\
0.65^{*}\end{array}$ & $1.19 \pm 0.22$ & $1.22 \pm 0.21$ & $1.34 \pm 0.34$ & $1.3 \pm 0.27$ \\
\hline & MT1M & metallothionein $1 \mathrm{M}$ & $\begin{array}{l}2.76 \pm \\
0.63^{*}\end{array}$ & $1.19 \pm 0.29$ & $1.25 \pm 0.19$ & $1.24 \pm 0.27$ & $1.18 \pm 0.31$ \\
\hline & MT1B & metallothionein 1B & $1.9 \pm 0.22^{*}$ & $1.08 \pm 0.17$ & $1.11 \pm 0.11$ & $1.16 \pm 0.2$ & $1.11 \pm 0.19$ \\
\hline & MT1X & metallothionein $1 \mathrm{X}$ & $\begin{array}{l}3.49 \pm \\
1.76^{*}\end{array}$ & $1.24 \pm 0.43$ & $1.33 \pm 0.4$ & $1.35 \pm 0.42$ & $1.28 \pm 0.49$ \\
\hline & MT1H & metallothionein $1 \mathrm{H}$ & $2.89 \pm 1.3^{*}$ & $1.27 \pm 0.34$ & $1.32 \pm 0.37$ & $1.3 \pm 0.31$ & $1.14 \pm 0.44$ \\
\hline & MT1G & metallothionein $1 \mathrm{G}$ & $\begin{array}{l}3.28 \pm \\
1.44^{*}\end{array}$ & $1.43 \pm 0.4$ & $1.48 \pm 0.53$ & $1.35 \pm 0.4$ & $1.12 \pm 0.41$ \\
\hline & SLC30A1 & $\begin{array}{l}\text { solute carrier family } 30 \\
\text { (zinc transporter). member } 1\end{array}$ & $\begin{array}{l}1.98 \pm \\
0.44^{*}\end{array}$ & $1.26 \pm 0.21$ & $1.11 \pm 0.12$ & $1.24 \pm 0.29$ & $1.24 \pm 0.25$ \\
\hline \multirow{6}{*}{$\begin{array}{l}\text { Aminoacyl-tRNA } \\
\text { biosynthesis }\end{array}$} & IARS & isoleucyl-tRNA synthetase & $1.15 \pm 0.18$ & $1.58 \pm 0.28^{*}$ & $1.25 \pm 0.29$ & $1.14 \pm 0.17$ & $1.22 \pm 0.17$ \\
\hline & AARS & alanyl-tRNA synthetase & $1.1 \pm 0.13$ & $1.41 \pm 0.21^{*}$ & $1.19 \pm 0.23$ & $1.04 \pm 0.14$ & $1.17 \pm 0.11$ \\
\hline & YARS & tyrosyl-tRNA synthetase & $1.24 \pm 0.15$ & $1.49 \pm 0.32^{*}$ & $1.28 \pm 0.24$ & $1.15 \pm 0.11$ & $1.21 \pm 0.13$ \\
\hline & MARS & methionyl-tRNA synthetase & $1.17 \pm 0.17$ & $1.62 \pm 0.33^{*}$ & $1.25 \pm 0.24$ & $1.12 \pm 0.14$ & $1.19 \pm 0.16$ \\
\hline & EPRS & glutamyl-prolyl-tRNA synthetase & $1.09 \pm 0.13$ & $1.37 \pm 0.12^{*}$ & $1.12 \pm 0.2$ & $1.09 \pm 0.19$ & $1.16 \pm 0.11$ \\
\hline & GARS & glycyl-tRNA synthetase & $1.3 \pm 0.18^{*}$ & $1.56 \pm 0.37^{*}$ & $1.38 \pm 0.22^{*}$ & $1.22 \pm 0.17$ & $1.3 \pm 0.16$ \\
\hline \multirow[t]{7}{*}{ PPAR signaling } & CPT1A & carnitine palmitoyltransferase $1 \mathrm{~A}$ & $1.25 \pm 0.1^{*}$ & $1.4 \pm 0.09^{*}$ & $1.32 \pm 0.18^{*}$ & $1.21 \pm 0.08$ & $1.2 \pm 0.12$ \\
\hline & SCD & $\begin{array}{l}\text { stearoyl-CoA desaturase } \\
\text { (delta-9-desaturase) }\end{array}$ & $1.03 \pm 0.09$ & $1.46 \pm 0.16^{*}$ & $1.56 \pm 0.22^{*}$ & $1.6 \pm 0.21^{*}$ & $2 \pm 0.33^{*}$ \\
\hline & ACSL1 & $\begin{array}{l}\text { acyl-CoA synthetase long-chain } \\
\text { family member } 1\end{array}$ & $1.3 \pm 0.1^{*}$ & $1.47 \pm 0.16^{*}$ & $1.51 \pm 0.15^{*}$ & $1.44 \pm 0.11^{*}$ & $1.2 \pm 0.19$ \\
\hline & ANGPTL4 & angiopoietin-like 4 & $1.4 \pm 0.19^{*}$ & $1.42 \pm 0.19^{*}$ & $1.47 \pm 0.33^{*}$ & $1.53 \pm 0.31^{*}$ & $1.37 \pm 0.26$ \\
\hline & ME1 & $\begin{array}{l}\text { malic enzyme 1, NADP(+)-dependent, } \\
\text { cytosolic }\end{array}$ & $1.15 \pm 0.13$ & $1.52 \pm 0.25^{*}$ & $1.47 \pm 0.31 *$ & $1.26 \pm 0.27$ & $1.52 \pm 0.32^{*}$ \\
\hline & SLC27A2 & $\begin{array}{l}\text { solute carrier family } 27 \\
\text { (fatty acid transporter), member } 2\end{array}$ & $1.25 \pm 0.14$ & $1.36 \pm 0.21 *$ & $1.27 \pm 0.2$ & $1.23 \pm 0.16$ & $1.61 \pm 0.4^{*}$ \\
\hline & GK & glycerol kinase & $1.04 \pm 0.09$ & $1.28 \pm 0.13^{*}$ & $1.34 \pm 0.24^{*}$ & $0.98 \pm 0.09$ & $0.95 \pm 0.13$ \\
\hline \multirow[t]{5}{*}{ Adipocytokine signaling } & CPT1A & carnitine palmitoyltransferase $1 \mathrm{~A}$ & $1.25 \pm 0.1^{*}$ & $1.4 \pm 0.09^{*}$ & $1.32 \pm 0.18^{*}$ & $1.21 \pm 0.08$ & $1.2 \pm 0.12$ \\
\hline & ACSL1 & $\begin{array}{l}\text { acyl-CoA synthetase long-chain } \\
\text { family member } 1\end{array}$ & $1.3 \pm 0.1^{*}$ & $1.47 \pm 0.16^{*}$ & $1.51 \pm 0.15^{*}$ & $1.44 \pm 0.11^{*}$ & $1.2 \pm 0.19$ \\
\hline & NFKBIA & $\begin{array}{l}\text { nuclear factor of kappa light } \\
\text { polypeptide gene enhancer in } \\
\text { B-cells inhibitor, alpha }\end{array}$ & $1.06 \pm 0.07$ & $1.33 \pm 0.03^{*}$ & $1.27 \pm 0.05^{*}$ & $1.39 \pm 0.22^{*}$ & $1.64 \pm 0.5$ \\
\hline & IRS2 & insulin receptor substrate 2 & $\begin{array}{l}1.54 \pm \\
0.24^{*}\end{array}$ & $1.49 \pm 0.25^{*}$ & $1.45 \pm 0.26^{*}$ & $1.48 \pm 0.2^{*}$ & $1.58 \pm 0.32^{*}$ \\
\hline & G6PC2 & glucose-6-phosphatase, Catalytic, 2 & $\begin{array}{l}1.27 \pm \\
0.06^{*}\end{array}$ & $1.32 \pm 0.14^{*}$ & $1.22 \pm 0.2$ & $1.12 \pm 0.13$ & $1.27 \pm 0.12$ \\
\hline \multirow[t]{2}{*}{ TNF signalling } & CXCL1 & $\begin{array}{l}\text { chemokine (C-X-C motif) ligand } 1 \\
\text { (melanoma growth stimulating } \\
\text { activity, alpha) }\end{array}$ & $0.87 \pm 0.11$ & $1.39 \pm 0.15^{*}$ & $1.58 \pm 0.19 *$ & $1.77 \pm 0.45^{*}$ & $1.43 \pm 0.29$ \\
\hline & PTGS2 & prostaglandin-endoperoxide synthase & $3.19 \pm$ & $2.1 \pm 0.36^{*}$ & $2.78 \pm 0.8^{*}$ & $2.72 \pm 0.26^{*}$ & $4.52 \pm 1.7$ \\
\hline
\end{tabular}


Table 1 Expression profile of genes from the enriched pathways over time (fold untreated \pm S.D., $n=5$ ) (Continued)

\begin{tabular}{|c|c|c|c|c|c|c|c|}
\hline Pathway name & $\begin{array}{l}\text { Gene } \\
\text { symbol }\end{array}$ & Gene name & $4 \mathrm{~h}$ & $12 \mathrm{~h}$ & $1 d$ & $2 d$ & $7 d$ \\
\hline & & $\begin{array}{l}2 \text { (prostaglandin G/H synthase and } \\
\text { cyclooxygenase) }\end{array}$ & $1.24^{*}$ & & & & \\
\hline & LIF & leukemia inhibitory factor & $1.05 \pm 0.08$ & $1.21 \pm 0.12$ & $1.3 \pm 0.1 *$ & $1.4 \pm 0.24^{*}$ & $1 \pm 0.04$ \\
\hline & $\mathrm{CXCL2}$ & chemokine (C-X-C motif) ligand 2 & $0.97 \pm 0.11$ & $1.3 \pm 0.16$ & $1.34 \pm 0.18^{*}$ & $1.5 \pm 0.34$ & $1.48 \pm 0.51$ \\
\hline & $\mathrm{BIRC} 3$ & baculoviral IAP repeat containing 3 & $0.93 \pm 0.1$ & $1.18 \pm 0.2$ & $1.3 \pm 0.19^{*}$ & $1.11 \pm 0.33$ & $0.82 \pm 0.3$ \\
\hline & ICAM1 & intercellular adhesion molecule 1 & $1.03 \pm 0.04$ & $1.24 \pm 0.16$ & $1.43 \pm 0.11^{*}$ & $1.64 \pm 0.32^{*}$ & $1.7 \pm 0.7$ \\
\hline & CX3CL1 & chemokine (C-X3-C motif) ligand 1 & $1.13 \pm 0.12$ & $1.23 \pm 0.18$ & $1.38 \pm 0.23 *$ & $1.59 \pm 0.68$ & $1.33 \pm 0.67$ \\
\hline & CCL20 & chemokine (C-C motif) ligand 20 & $0.76 \pm 0.3$ & $1.57 \pm 0.63$ & $2.5 \pm 0.9^{*}$ & $1.61 \pm 0.32^{*}$ & $1.28 \pm 0.4$ \\
\hline \multirow[t]{18}{*}{$\begin{array}{l}\text { Metabolism of } \\
\text { xenobiotics by P450 }\end{array}$} & CYP2C9 & $\begin{array}{l}\text { cytochrome P450, family } 2, \\
\text { subfamily C, polypeptide } 9\end{array}$ & $0.87 \pm 0.05$ & $0.86 \pm 0.1$ & $0.8 \pm 0.06$ & $0.73 \pm 0.11^{*}$ & $0.62 \pm 0.12^{*}$ \\
\hline & CYP1A1 & $\begin{array}{l}\text { cytochrome P450, family } 1, \\
\text { subfamily A, polypeptide } 1\end{array}$ & $1.23 \pm 0.43$ & $1 \pm 0.34$ & $0.7 \pm 0.13^{*}$ & $0.6 \pm 0.24^{*}$ & $0.35 \pm 0.09^{*}$ \\
\hline & CYP1B1 & $\begin{array}{l}\text { cytochrome P450, family } 1 \text {, } \\
\text { subfamily B, polypeptide } 1\end{array}$ & $1.14 \pm 0.22$ & $1.13 \pm 0.23$ & $0.87 \pm 0.16$ & $0.89 \pm 0.22$ & $0.64 \pm 0.17^{*}$ \\
\hline & UGT2B7 & $\begin{array}{l}\text { UDP glucuronosyltransferase } \\
2 \text { family, polypeptide B7 }\end{array}$ & $0.91 \pm 0.15$ & $0.86 \pm 0.11$ & $0.79 \pm 0.06$ & $0.77 \pm 0.1^{*}$ & $0.64 \pm 0.06^{*}$ \\
\hline & UGT2A3 & $\begin{array}{l}\text { UDP glucuronosyltransferase } \\
2 \text { family, polypeptide A3 }\end{array}$ & $0.95 \pm 0.2$ & $0.96 \pm 0.22$ & $0.71 \pm 0.18^{*}$ & $0.59 \pm 0.13^{*}$ & $0.29 \pm 0.06^{*}$ \\
\hline & GSTA1 & glutathione S-transferase alpha 1 & $1.1 \pm 0.2$ & $1.11 \pm 0.23$ & $0.98 \pm 0.2$ & $0.73 \pm 0.16^{*}$ & $0.45 \pm 0.08^{*}$ \\
\hline & GSTA2 & glutathione S-transferase alpha 2 & $1.1 \pm 0.24$ & $1.16 \pm 0.31$ & $0.97 \pm 0.23$ & $0.68 \pm 0.2^{*}$ & $0.36 \pm 0.06^{*}$ \\
\hline & UGT2B15 & $\begin{array}{l}\text { UDP glucuronosyltransferase } \\
2 \text { family, polypeptide B15 }\end{array}$ & $0.89 \pm 0.22$ & $0.89 \pm 0.27$ & $0.53 \pm 0.12^{*}$ & $0.37 \pm 0.13^{*}$ & $0.16 \pm 0.03^{*}$ \\
\hline & UGT2B17 & $\begin{array}{l}\text { UDP glucuronosyltransferase } \\
2 \text { family, polypeptide B17 }\end{array}$ & $0.87 \pm 0.23$ & $0.88 \pm 0.25$ & $0.58 \pm 0.11 *$ & $0.43 \pm 0.15^{*}$ & $0.23 \pm 0.06^{*}$ \\
\hline & UGT2B10 & $\begin{array}{l}\text { UDP glucuronosyltransferase } \\
2 \text { family, polypeptide B10 }\end{array}$ & $0.97 \pm 0.07$ & $0.98 \pm 0.11$ & $0.96 \pm 0.1$ & $0.94 \pm 0.09$ & $0.77 \pm 0.09^{*}$ \\
\hline & $\mathrm{ADH} 1 \mathrm{~B}$ & $\begin{array}{l}\text { alcohol dehydrogenase } 1 \mathrm{~B} \\
\text { (class I), beta polypeptide }\end{array}$ & $0.86 \pm 0.1$ & $0.82 \pm 0.1$ & $0.74 \pm 0.11^{*}$ & $0.69 \pm 0.13^{*}$ & $0.6 \pm 0.11^{*}$ \\
\hline & $\mathrm{ADH} 1 \mathrm{~A}$ & $\begin{array}{l}\text { alcohol dehydrogenase } 1 \mathrm{~A} \text { (class I), } \\
\text { alpha polypeptide }\end{array}$ & $0.86 \pm 0.07$ & $0.87 \pm 0.1$ & $0.73 \pm 0.15^{*}$ & $0.67 \pm 0.16^{*}$ & $0.58 \pm 0.17^{*}$ \\
\hline & $\mathrm{ADH} 1 \mathrm{C}$ & $\begin{array}{l}\text { alcohol dehydrogenase } 1 \text { C (class I), } \\
\text { gamma polypeptide }\end{array}$ & $\begin{array}{l}0.76 \pm \\
0.13^{*}\end{array}$ & $0.65 \pm 0.17^{*}$ & $0.5 \pm 0.18^{*}$ & $0.39 \pm 0.14^{*}$ & $0.17 \pm 0.08^{*}$ \\
\hline & CYP3A4 & $\begin{array}{l}\text { cytochrome P450, family } 3, \\
\text { subfamily A, polypeptide } 4\end{array}$ & $0.83 \pm 0.11$ & $1 \pm 0.02$ & $0.72 \pm 0.16^{*}$ & $0.57 \pm 0.17^{*}$ & $0.55 \pm 0.23^{*}$ \\
\hline & CYP3A5 & $\begin{array}{l}\text { cytochrome P450, family } 3, \\
\text { subfamily A, polypeptide } 5\end{array}$ & $0.94 \pm 0.14$ & $0.97 \pm 0.14$ & $0.82 \pm 0.1$ & $0.68 \pm 0.15^{*}$ & $0.36 \pm 0.07^{*}$ \\
\hline & AKR1C1 & $\begin{array}{l}\text { aldo-keto reductase family } 1 \text {, } \\
\text { member } \mathrm{C} 1\end{array}$ & $1.05 \pm 0.11$ & $1.32 \pm 0.13^{*}$ & $1.3 \pm 0.22$ & $0.95 \pm 0.07$ & $0.62 \pm 0.08^{*}$ \\
\hline & AKR1C2 & $\begin{array}{l}\text { aldo-keto reductase family } 1 \text {, } \\
\text { member } C 2\end{array}$ & $1.1 \pm 0.12$ & $1.36 \pm 0.16^{*}$ & $1.34 \pm 0.23$ & $0.95 \pm 0.08$ & $0.62 \pm 0.1^{*}$ \\
\hline & $\mathrm{ALDH} 1 \mathrm{~A} 3$ & $\begin{array}{l}\text { aldehyde dehydrogenase } \\
1 \text { family, member } \mathrm{A} 3\end{array}$ & $0.96 \pm 0.08$ & $1.03 \pm 0.11$ & $0.92 \pm 0.08$ & $0.9 \pm 0.05$ & $0.64 \pm 0.17^{*}$ \\
\hline \multirow[t]{5}{*}{$\begin{array}{l}\text { Glycolysis/ } \\
\text { gluconeogenesis }\end{array}$} & ALDH3A2 & $\begin{array}{l}\text { aldehyde dehydrogenase } 3 \\
\text { family, member A2 }\end{array}$ & $1 \pm 0.14$ & $1.1 \pm 0.19$ & $0.96 \pm 0.16$ & $0.84 \pm 0.12$ & $0.71 \pm 0.11^{*}$ \\
\hline & G6PC & $\begin{array}{l}\text { glucose-6-phosphatase. } \\
\text { Catalytic subunit }\end{array}$ & $1.29 \pm 0.33$ & $0.9 \pm 0.3$ & $0.74 \pm 0.17^{*}$ & $0.66 \pm 0.16^{*}$ & $0.46 \pm 0.12^{*}$ \\
\hline & $\mathrm{ADH} 1 \mathrm{~B}$ & $\begin{array}{l}\text { alcohol dehydrogenase } 1 \mathrm{~B} \\
\text { (class I), beta polypeptide }\end{array}$ & $0.86 \pm 0.1$ & $0.82 \pm 0.1$ & $0.74 \pm 0.11 *$ & $0.69 \pm 0.13^{*}$ & $0.6 \pm 0.11^{*}$ \\
\hline & $\mathrm{ADH} 1 \mathrm{C}$ & $\begin{array}{l}\text { alcohol dehydrogenase } 1 C \\
\text { (class I), gamma polypeptide }\end{array}$ & $\begin{array}{l}0.76 \pm \\
0.13^{*}\end{array}$ & $0.65 \pm 0.17^{*}$ & $0.5 \pm 0.18^{*}$ & $0.39 \pm 0.14^{*}$ & $0.17 \pm 0.08^{*}$ \\
\hline & FBP1 & fructose-1.6-bisphosphatase 1 & $0.97 \pm 0.07$ & $0.97 \pm 0.1$ & $0.94 \pm 0.04$ & $0.9 \pm 0.06$ & $0.74 \pm 0.07^{*}$ \\
\hline
\end{tabular}


Table 1 Expression profile of genes from the enriched pathways over time (fold untreated \pm S.D., $n=5$ ) (Continued)

\begin{tabular}{|c|c|c|c|c|c|c|c|}
\hline Pathway name & $\begin{array}{l}\text { Gene } \\
\text { symbol }\end{array}$ & Gene name & $4 \mathrm{~h}$ & $12 \mathrm{~h}$ & $1 d$ & $2 d$ & $7 d$ \\
\hline & $\mathrm{ADH} 1 \mathrm{~A}$ & $\begin{array}{l}\text { alcohol dehydrogenase } 1 \mathrm{~A} \\
\text { (class I), alpha polypeptide }\end{array}$ & $0.86 \pm 0.07$ & $0.87 \pm 0.1$ & $0.73 \pm 0.15^{*}$ & $0.67 \pm 0.16^{*}$ & $0.58 \pm 0.17^{*}$ \\
\hline & ALDOB & aldolase B, fructose-bisphosphate & $0.86 \pm 0.19$ & $0.79 \pm 0.2$ & $0.67 \pm 0.14^{*}$ & $0.37 \pm 0.18^{*}$ & $0.14 \pm 0.06$ \\
\hline & ALDH1A3 & $\begin{array}{l}\text { aldehyde dehydrogenase } 1 \text { family, } \\
\text { member } \mathrm{A} 3\end{array}$ & $0.96 \pm 0.08$ & $1.03 \pm 0.11$ & $0.92 \pm 0.08$ & $0.9 \pm 0.05$ & $0.64 \pm 0.17^{*}$ \\
\hline \multirow[t]{6}{*}{ Fatty acid degradation } & ALDH3A2 & $\begin{array}{l}\text { aldehyde dehydrogenase } 3 \text { family, } \\
\text { member A2 }\end{array}$ & $1 \pm 0.14$ & $1.1 \pm 0.19$ & $0.96 \pm 0.16$ & $0.84 \pm 0.12$ & $0.71 \pm 0.11^{*}$ \\
\hline & ACSL1 & $\begin{array}{l}\text { acyl-CoA synthetase long-chain } \\
\text { family member } 1\end{array}$ & $1.3 \pm 0.1^{*}$ & $1.47 \pm 0.16^{*}$ & $1.51 \pm 0.15^{*}$ & $1.44 \pm 0.11^{*}$ & $1.2 \pm 0.19$ \\
\hline & ACSL5 & $\begin{array}{l}\text { acyl-CoA synthetase long-chain } \\
\text { family member } 5\end{array}$ & $1.04 \pm 0.08$ & $1.16 \pm 0.06$ & $1.19 \pm 0.22$ & $0.97 \pm 0.15$ & $0.76 \pm 0.13^{*}$ \\
\hline & $\mathrm{ADH} 1 \mathrm{C}$ & $\begin{array}{l}\text { alcohol dehydrogenase } 1 C \text { (class I), } \\
\text { gamma polypeptide }\end{array}$ & $\begin{array}{l}0.76 \pm \\
0.13^{*}\end{array}$ & $0.65 \pm 0.17^{*}$ & $0.5 \pm 0.18^{*}$ & $0.39 \pm 0.14^{*}$ & $0.17 \pm 0.08^{*}$ \\
\hline & $\mathrm{ADH} 1 \mathrm{~B}$ & $\begin{array}{l}\text { alcohol dehydrogenase 1B (class I), } \\
\text { beta polypeptide }\end{array}$ & $0.86 \pm 0.1$ & $0.82 \pm 0.1$ & $0.74 \pm 0.11 *$ & $0.69 \pm 0.13^{*}$ & $0.6 \pm 0.11^{*}$ \\
\hline & ADH1A & $\begin{array}{l}\text { alcohol dehydrogenase } 1 \mathrm{~A} \text { (class I), } \\
\text { alpha polypeptide }\end{array}$ & $0.86 \pm 0.07$ & $0.87 \pm 0.1$ & $0.73 \pm 0.15^{*}$ & $0.67 \pm 0.16^{*}$ & $0.58 \pm 0.17^{*}$ \\
\hline
\end{tabular}

*Indicates DEGs. Bold indicates the exposure time when the pathway was enriched

important role in palmitate-induced dysfunction of isolated human islets [24]. In our previous study, protein profiling of palmitate-treated INS-1E cells by using 2D gel electrophoresis and MALDI-TOF MS identified 31 differentially expressed proteins with a function in carbohydrate or protein metabolism and $\mathrm{Ca}^{2+}$ or mRNA binding [25]. In a similar study, Maris and co-authors identified 83 differentially expressed proteins with a function in ER stress, insulin maturation, intracellular trafficking and generation of harmful metabolites and reactive oxygen species [26].

In the current study, the transcriptomics approach identified 903 DEGs in palmitate-treated isolated human islets. The lower number of DEGs compared with previous studies is due to cut-off that was applied for DEGs: 1.3-fold change after palmitate exposure compared to untreated islets. Of identified genes, 30\% were differentially expressed prior to failure of the human islets (up to 1 day of palmitate exposure) and belonged to 8 enriched pathways. These findings underline the importance of understanding early biological changes in islets upon palmitate treatment.

\section{Analysis of pathways enriched prior to decline of islet function}

Four of the eight identified pathways enriched prior to decline of beta-cell function were not enriched in islets with declining function. One pathway of the eight pathways was enriched only when insulin hypersecretion was most prominent. These pathways are discussed in more detail.

The "Mineral absorption" pathway was enriched after 4-h exposure but not at any later time point. The pathway was predicted based on 9 transcripts encoding different subtypes of metallothionein (MT) gene and 1 transcript encoding a solute carrier family 30 (zinc transporter) (Table 1). All the transcripts were elevated in the presence of palmitate. MTs regulate the intracellular level of free zinc, which is known to be essential for the primary functions of beta-cells including insulin biosynthesis and insulin storage. Dysregulation or dysfunction of zinc-transporting proteins leads to impairment of insulin processing and glucose metabolism [27, 28]. Polymorphisms in genes encoding MTs have been related to the development of T2DM [29]. Transgenic mice, with a beta-cell-specific over-expression of MT-2 displayed a significantly reduced beta-cell death and a better preserved insulin production when exposed to streptozotocin [30]. Also, addition of extracellular Zn7-MT-2A potentiated insulin production and secretion from insulin-producing INS-1E beta-cell culture [31]. The abovementioned suggest that an initial increase in MTs transcript level in palmitate-treated human islets is an adaptive mechanism to support insulin synthesis during the insulin hypersecretion.

Another mechanism with a potentially adaptive role is the "Aminoacyl-tRNA biosynthesis" pathway. The pathway was predicted based on 6 tRNA synthetases elevated after $12 \mathrm{~h}$ of palmitate exposure (Table 1). Presumably, increased levels of tRNA synthetases enhance insulin biosynthesis, which allows to maintain insulin hypersecretion from palmitate-treated islets. However, due to a short-term up-regulation of these genes, upon longer exposure to palmitate the rate of insulin synthesis falls, causing degranulation of human islets. In obese individuals, hypersecretion of insulin compensates insulin resistance in peripheral tissues caused by elevated levels of 
circulating fatty acids. Exhaustion of islets would lead to inability to secrete sufficient insulin to compensate insulin resistance and would trigger the development of T2DM.

The "PPAR signalling" and "Adipocytokine signalling" pathways were enriched after 12-h and 1-day exposure to palmitate and included genes with a function in fatty acid and glucose metabolism (Table 1). All the genes from these pathways were elevated. Palmitate-induced upregulation of the genes involved in fatty acid metabolism has been also shown in previous transcriptomics studies [22, 23]. Considering that increased metabolism and beta-oxidation of fatty acids are beneficial for beta cells [32, 33], activation of these pathways is, apparently, an adaptive response to fatty acid exposure. This is supported by previous reports about the protective role of PPAR1 pathway in palmitate-treated beta cells [34]. Although "PPAR signalling" and "Adipocytokine signalling" pathways are not among the enriched pathways after 2and 7-day exposure to palmitate, most of the genes from these pathways remained at elevated levels (Table 1). It suggests that the contribution of these genes and pathways to beta-cell biology becomes minor and cannot combat the deleterious pathways activated in beta cells upon the long-term exposure to palmitate.

In summary, all the pathways enriched prior to but not during the decline of islet function play an adaptive/ protective role in fatty acid-treated human islets.

The "TNF signalling pathway" was predicted based on 8 genes elevated after 1- and 2-day exposure to palmitate (Table 1). Most transcripts in "TNF signalling" pathway encode chemokines. It is known that pancreatic islets produce and secrete a variety of chemokines [35]. In patients, these chemokines may recruit leukocytes into pancreatic tissue and cause beta-cell dysfunction and destruction [35]. However, such inflammatory response is unlikely in our system with isolated islets. Instead, chemokines may interact with numerous chemokine receptors expressed on human islets and, in such way, trigger pathway signalling [36, 37]. The mechanism of chemokine-induced signalling and its effect on insulin secretion is poorly investigated [38]. Considering that activation of "TNF signalling pathway" coincides with insulin hypersecretion one may speculate that this pathway contributes to hypersecretion of insulin rather than exerts direct protective or deleterious effects.

\section{Analysis of pathways enriched both prior to and during decline of islet function}

Three pathways were enriched after 1, 2 and 7 days of palmitate exposure i.e. both prior and during the decline of islet function. The "Fatty acid degradation" and "Glycolysis/gluconeogenesis" pathways were not among the top pathways and were mainly based on the same list of genes as "Metabolism of xenobiotics by $\mathrm{P} 450$ " pathway (Table 1). Therefore, we will discuss the "Metabolism of xenobiotics by $\mathrm{P} 450$ " pathway, which was the top pathway after 1 and 2 days and the second top pathway after 7 days of palmitate exposure (Fig. 4).

The "Metabolism of xenobiotics by P450" pathway was predicted based on the reduced expression of genes encoding the detoxification enzymes (Table 1). These enzymes convert drugs and xenobiotics into water-soluble metabolites and play a central role in their detoxification [39]. Elimination of xenobiotics predominantly occurs in liver, kidneys and gastrointestinal tract. However, other tissues/organs are also equipped with diverse detoxification enzymes to minimize the insults caused by xenobiotics [40].

In addition, detoxification enzymes play a role in metabolism of fatty acids via $\omega$-oxidation, a process almost identical to xenobiotic detoxification [41, 42]. During $\omega$-oxidation, which takes place in ER, mixed-function oxidases (CYPs), alcohol dehydrogenases and aldehyde dehydrogenases convert fatty acids into dicarboxylic acid [42]. The formed dicarboxylic acid enters mitochondria or peroxisomes for further metabolism via beta-oxidation [43]. It has been proposed that $\omega$-oxidation is a rescue pathway that allows to eliminate toxic levels of fatty acids that accumulate in the cells when the main $\beta$-oxidation pathway is overwhelmed [44]. CYPs that are known to contribute to $\omega$-oxidation is of class 4 [45]. However, it does not rule out that CYP proteins of class 1,2 and 3, identified in our study, also catalyse $\omega$-oxidation considering that CYPs possess broad substrate specificities [42, 46]. The contribution of glutathione S-transferases and UDP-glucuronosyltransferases to fatty acid metabolism is not known.

Since the detoxification enzymes are important for detoxification of fatty acids, we assume that the inhibition of the "Metabolism of xenobiotics by P450" pathway in human islets upon palmitate exposure is an early deleterious event. It causes accumulation of toxic amounts of fatty acids which contributes to a failure of the islets.

\section{Conclusion}

We propose that in palmitate-treated human islets, at early time points, protective events, including up-regulation of metallothioneins, tRNA synthetases and fatty acid-metabolising proteins, dominate over deleterious events, including inhibition of fatty acid detoxification enzymes, which contributes to potentiation of GSIS. After prolonged exposure of islets to palmitate, the protective events are outweighed by the deleterious events, which contribute to impaired GSIS. The study identifies temporal order between different cellular events, which either promote or protect from beta-cell failure. The sequence of these events should be considered when developing strategies for prevention and treatment of the disease. 


\section{Additional files}

Additional file 1: Table S1. List of pathways enriched at least at one culture time point. (DOC $63 \mathrm{~kb}$ )

Additional file 2: Table S2. List of enriched pathways after $4 \mathrm{~h}$ of palmitate treatment. (DOC $28 \mathrm{~kb}$ )

Additional file 3: Table S3. List of enriched pathways after $12 \mathrm{~h}$ of palmitate treatment. (DOC $32 \mathrm{~kb}$ )

Additional file 4: Table S4. List of enriched pathways after 1 day of palmitate treatment. (DOC $37 \mathrm{~kb}$ )

Additional file 5: Table S5. List of enriched pathways after 2 days of palmitate treatment. (DOC $49 \mathrm{~kb}$ )

Additional file 6: Table S6. List of enriched pathways after 7 days of palmitate treatment. (DOC $53 \mathrm{~kb}$ )

\section{Abbreviations}

BSA: bovine serum albumin; DEGs: differentially expressed genes; DPBS: Dulbecco's phosphate buffered saline; FFA: free fatty acids; GSIS: glucose-stimulated insulin secretion; HTA: Human Transcriptome Array; T2DM: type 2 diabetes mellitus

\section{Acknowledgements}

We would like to acknowledge Array and Analysis Facility, Science for Life Laboratory at Uppsala Biomedical Center (BMC), Uppsala University for their help with Affymetrix microarray analysis.

\section{Funding}

The work was supported by the European Commission FP7 Project BeatJudo (grant number 279 153).

\section{Availability of data and materials}

Microarray data from HTA 2.0 are deposited and available at Gene Expression Omnibus (GEO) (https://www.ncbi.nlm.nih.gov/geo/query/

acc.cgi?acc=GSE1 18230)

\section{Authors' contributions}

ES and PB designed the research, ES and JC performed the experiments, ES KR and RS performed the bioinformatics analysis, ES analysed and interpreted the data and wrote the manuscript, PB and RS obtained the research funding. All authors read, commented and approved the final version of the manuscript.

\section{Ethics approval and consent to participate}

Ethical permission to use human islets has been obtained from the Regional Ethical Review Board in Uppsala, Sweden (EPN number 2010/006; 2010-02-10).

\section{Consent for publication}

Not applicable.

\section{Competing interests}

The authors declare that they have no competing interests.

\section{Publisher's Note}

Springer Nature remains neutral with regard to jurisdictional claims in published maps and institutional affiliations.

\section{Author details}

${ }^{1}$ Department of Medical Cell Biology, Uppsala University, Box 571, 75123 Uppsala, Sweden. ${ }^{2}$ Molecular Neuroscience Group, Institute of Molecular Biology, National Academy of Sciences, 0014 Yerevan, Armenia.

${ }^{3}$ Luxembourg Centre for Systems Biomedicine, University of Luxembourg Campus Belval, 7 avenue des Hauts fourneaux, 4362 Esch-Belval, Luxembourg City, Luxembourg.
Received: 17 March 2018 Accepted: 14 August 2018

Published online: 22 August 2018

\section{References}

1. Staaf J, Ubhayasekera SJ, Sargsyan E, Chowdhury A, Kristinsson H, Manell H, Bergquist J, Forslund A, Bergsten P. Initial hyperinsulinemia and subsequent beta-cell dysfunction is associated with elevated palmitate levels. Pediatr Res. 2016

2. Le Stunff C, Bougneres P. Early changes in postprandial insulin secretion, not in insulin sensitivity, characterize juvenile obesity. Diabetes. 1994;43(5): 696-702.

3. Weyer C, Hanson RL, Tataranni PA, Bogardus C, Pratley RE. A high fasting plasma insulin concentration predicts type 2 diabetes independent of insulin resistance: evidence for a pathogenic role of relative hyperinsulinemia. Diabetes. 2000;49(12):2094-101.

4. Kristinsson H, Smith DM, Bergsten P, Sargsyan E. FFAR1 is involved in both the acute and chronic effects of palmitate on insulin secretion. Endocrinology. 2013;154(11):4078-88.

5. Stein DT, Stevenson BE, Chester MW, Basit M, Daniels MB, Turley SD, McGarry JD. The insulinotropic potency of fatty acids is influenced profoundly by their chain length and degree of saturation. J Clin Invest. 1997:100(2):398-403.

6. Zhou YP, Grill V. Long term exposure to fatty acids and ketones inhibits Bcell functions in human pancreatic islets of Langerhans. J Clin Endocrinol Metab. 1995;80(5):1584-90.

7. Clark CM Jr, Lee DA. Prevention and treatment of the complications of diabetes mellitus. N Engl J Med. 1995;332(18):1210-7.

8. Boucher A, Lu D, Burgess SC, Telemaque-Potts S, Jensen MV, Mulder H, Wang MY, Unger RH, Sherry AD, Newgard CB. Biochemical mechanism of lipid-induced impairment of glucose-stimulated insulin secretion and reversal with a malate analogue. J Biol Chem. 2004;279(26):27263-71.

9. Kristinsson H, Bergsten P, Sargsyan E. Free fatty acid receptor 1 (FFAR1/ GPR40) signaling affects insulin secretion by enhancing mitochondrial respiration during palmitate exposure. Biochim Biophys Acta. 2015;1853(12): 3248-57.

10. Sargsyan E, Sol ER, Bergsten P. UPR in palmitate-treated pancreatic betacells is not affected by altering oxidation of the fatty acid. Nutrition \& metabolism. 2011;8:70

11. Lupi R, Dotta F, Marselli L, Del Guerra S, Masini M, Santangelo C, Patane G, Boggi U, Piro S, Anello M, et al. Prolonged exposure to free fatty acids has cytostatic and pro-apoptotic effects on human pancreatic islets: evidence that beta-cell death is caspase mediated, partially dependent on ceramide pathway, and BCl-2 regulated. Diabetes. 2002;51(5):1437-42.

12. Manukyan L, Ubhayasekera SJ, Bergquist J, Sargsyan E, Bergsten P. Palmitate-induced impairments of beta-cell function are linked with generation of specific ceramide species via acylation of sphingosine. Endocrinology. 2015;156(3):802-12.

13. Hoppa MB, Collins S, Ramracheya R, Hodson L, Amisten S, Zhang Q, Johnson P, Ashcroft FM, Rorsman P. Chronic palmitate exposure inhibits insulin secretion by dissociation of $\mathrm{Ca}(2+)$ channels from secretory granules. Cell Metab. 2009;10(6):455-65.

14. Wu J, Sun $P$, Zhang $X$, Liu $H$, Jiang $H$, Zhu W, Wang $H$, Inhibition of GPR40 protects MIN6 beta cells from palmitate-induced ER stress and apoptosis. J Cell Biochem. 2012;113(4):1152-8.

15. Bergsten P, Hellman B. Glucose-induced amplitude regulation of pulsatile insulin secretion from individual pancreatic islets. Diabetes. 1993:42(5):670-4.

16. Irizarry RA, Hobbs B, Collin F, Beazer-Barclay YD, Antonellis KJ, Scherf U, Speed TP. Exploration, normalization, and summaries of high density oligonucleotide array probe level data. Biostatistics. 2003:4(2):249-64.

17. Li C, Wong WH. Model-based analysis of oligonucleotide arrays: expression index computation and outlier detection. Proc Natl Acad Sci U S A. 2001. 98(1):31-6.

18. Kamburov A, Stelzl U, Lehrach H, Herwig R. The ConsensusPathDB interaction database: 2013 update. Nucleic Acids Res. 2013;41(Database issue):D793-800

19. Kamburov A, Wierling C, Lehrach H, Herwig R. ConsensusPathDB--a database for integrating human functional interaction networks. Nucleic Acids Res. 2009;37(Database issue):D623-8.

20. Henquin JC, Ravier MA, Nenquin M, Jonas JC, Gilon P. Hierarchy of the betacell signals controlling insulin secretion. Eur J Clin Investig. 2003;33(9):742-50. 
21. White MG, Shaw JA, Taylor R. Type 2 diabetes: the pathologic basis of reversible beta-cell dysfunction. Diabetes Care. 2016;39(11):2080-8.

22. Cnop M, Abdulkarim B, Bottu G, Cunha DA, Igoillo-Esteve M, Masini M, Turatsinze JV, Griebel T, Villate O, Santin I, et al. RNA sequencing identifies dysregulation of the human pancreatic islet transcriptome by the saturated fatty acid palmitate. Diabetes. 2014;63(6):1978-93.

23. Hall E, Volkov P, Dayeh T, Bacos K, Ronn T, Nitert MD, Ling C. Effects of palmitate on genome-wide mRNA expression and DNA methylation patterns in human pancreatic islets. BMC Med. 2014;12:103.

24. Roomp K, Kristinsson H, Schvartz D, Ubhayasekera K, Sargsyan E, Manukyan L, Chowdhury A, Manell H, Satagopam V, Groebe K, et al. Combined lipidomic and proteomic analysis of isolated human islets exposed to palmitate reveals time-dependent changes in insulin secretion and lipid metabolism. PLoS One. 2017;12(4):e0176391.

25. Hovsepyan M, Sargsyan E, Bergsten P. Palmitate-induced changes in protein expression of insulin secreting INS-1E cells. J Proteome. 2010;73(6):1148-55.

26. Maris M, Robert S, Waelkens E, Derua R, Hernangomez MH, D'Hertog W, Cnop M, Mathieu C, Overbergh L. Role of the saturated nonesterified fatty acid palmitate in beta cell dysfunction. J Proteome Res. 2013;12(1):347-62

27. Mocchegiani E, Giacconi R, Malavolta M. Zinc signalling and subcellular distribution: emerging targets in type 2 diabetes. Trends Mol Med. 2008; 14(10):419-28.

28. Smidt $K$, Jessen $N$, Petersen AB, Larsen A, Magnusson N, Jeppesen JB, Stoltenberg M, Culvenor JG, Tsatsanis A, Brock B, et al. SLC30A3 responds to glucose- and zinc variations in beta-cells and is critical for insulin production and in vivo glucose-metabolism during beta-cell stress. PLoS One. 2009:4(5):e5684.

29. Yang L, Li H, Yu T, Zhao H, Cherian MG, Cai L, Liu Y. Polymorphisms in metallothionein- 1 and -2 genes associated with the risk of type 2 diabetes mellitus and its complications. Am J Physiol Endocrinol Metab. 2008;294(5):E987-92

30. Chen H, Carlson EC, Pellet L, Moritz JT, Epstein PN. Overexpression of metallothionein in pancreatic beta-cells reduces streptozotocin-induced DNA damage and diabetes. Diabetes. 2001;50(9):2040-6.

31. Nygaard SB, Lund NS, Larsen A, Pedersen N, Rungby J, Smidt K. Exogenous metallothionein potentiates the insulin response at normal glucose concentrations in INS-1E beta-cells without disturbing intracellular ZnT8 expression. Basic Clin Pharmacol Toxicol. 2015;116(2):173-7.

32. Cnop M, Hannaert JC, Hoorens A, Eizirik DL, Pipeleers DG. Inverse relationship between cytotoxicity of free fatty acids in pancreatic islet cells and cellular triglyceride accumulation. Diabetes. 2001;50(8):1771-7.

33. Sol EM, Sargsyan E, Akusjarvi G, Bergsten P. Glucolipotoxicity in INS-1E cells is counteracted by carnitine palmitoyltransferase 1 over-expression. Biochem Biophys Res Commun. 2008;375(4):517-21.

34. Hellemans K, Kerckhofs K, Hannaert JC, Martens G, Van Veldhoven P, Pipeleers D. Peroxisome proliferator-activated receptor alpha-retinoid $X$ receptor agonists induce beta-cell protection against palmitate toxicity. FEBS J. 2007;274(23):6094-105.

35. Sarkar SA, Lee CE, Victorino F, Nguyen TT, Walters JA, Burrack A, Eberlein J, Hildemann SK, Homann D. Expression and regulation of chemokines in murine and human type 1 diabetes. Diabetes. 2012;61 (2):436-46.

36. Collier JJ, Sparer TE, Karlstad MD, Burke SJ. Pancreatic islet inflammation: an emerging role for chemokines. J Mol Endocrinol. 2017;59(1):R33-46.

37. Zweemer AJ, Toraskar J, Heitman LH, AP IJ. Bias in chemokine receptor signalling. Trends Immunol. 2014;35(6):243-52.

38. Amisten S, Salehi A, Rorsman P, Jones PM, Persaud SJ. An atlas and functional analysis of $\mathrm{G}$-protein coupled receptors in human islets of Langerhans. Pharmacol Ther. 2013;139(3):359-91.

39. Mackenzie PI, Somogyi AA, Miners JO. Advances in drug metabolism and pharmacogenetics research in Australia. Pharmacol Res. 2017;116:7-19.

40. Rushmore TH, Kong AN. Pharmacogenomics, regulation and signaling pathways of phase I and II drug metabolizing enzymes. Curr Drug Metab. 2002;3(5):481-90.

41. Bishop-Bailey D, Thomson S, Askari A, Faulkner A, Wheeler-Jones C. Lipidmetabolizing CYPs in the regulation and dysregulation of metabolism. Annu Rev Nutr. 2014;34:261-79.

42. Miura Y. The biological significance of omega-oxidation of fatty acids. Proceedings of the Japan Academy Series B, Physical and biological sciences. 2013;89(8):370-82

43. Ferdinandusse S, Denis S, Van Roermund CW, Wanders RJ, Dacremont G. Identification of the peroxisomal beta-oxidation enzymes involved in the degradation of long-chain dicarboxylic acids. J Lipid Res. 2004;45(6):1104-11.
44. Wanders RJ, Komen J, Kemp S. Fatty acid omega-oxidation as a rescue pathway for fatty acid oxidation disorders in humans. FEBS J. 2011;278(2): 182-94.

45. Hardwick JP. Cytochrome P450 omega hydroxylase (CYP4) function in fatty acid metabolism and metabolic diseases. Biochem Pharmacol. 2008;75(12): 2263-75.

46. Zanger UM, Schwab M. Cytochrome P450 enzymes in drug metabolism: regulation of gene expression, enzyme activities, and impact of genetic variation. Pharmacol Ther. 2013:138(1):103-41.
Ready to submit your research? Choose BMC and benefit from:

- fast, convenient online submission

- thorough peer review by experienced researchers in your field

- rapid publication on acceptance

- support for research data, including large and complex data types

- gold Open Access which fosters wider collaboration and increased citations

- maximum visibility for your research: over $100 \mathrm{M}$ website views per year

At BMC, research is always in progress.

Learn more biomedcentral.com/submissions 